MIMS Technical Report No.00012 (200806092)

\title{
RAY-SINGER ZETA FUNCTIONS FOR COMPACT FLAT MANIFOLDS
}

\author{
TOSHIKAZU SUNADA AND HAJIME URAKAWA
}

\section{INTRODUCTION}

Let $M$ be a compact oriented Riemannian manifold, and let $\rho: \pi_{1}(M) \longrightarrow U(N)$ be a representation of the fundamental group of $M$ by unitary matrices. We denote by $E_{\rho}$ the flat vector bundle associated with $\rho$, and by $\Delta_{p}^{\rho}$ the Laplacian acting on $E_{\rho}$-valued $p$-forms on $M$. The Ray-Singer zeta function is defined by

$$
Z_{\rho}(s)=\sum_{p=0}^{n}(-1)^{p} p \zeta_{p}(s)
$$

where

$$
\zeta_{p}(s)=\Gamma(s)^{-1} \int_{0}^{\infty} t^{s-1}\left[\operatorname{tr}\left(e^{-t \Delta_{p}^{\rho}}\right)-\operatorname{dim} \operatorname{Ker} \Delta_{p}^{\rho}\right] d t .
$$

We shall show that $Z_{\rho}(s)$ for a compact flat manifold is expressed in terms of the Hurwits zeta function

$$
\zeta(s, \theta)=\sum_{n=0}^{\infty}(n+\theta)^{-s} \quad(0<\theta \leq 1)
$$

The number $\theta$ appearing in the expression turns out to be closely connected with a certain class of closed geodesics. We may in particular evaluate the value $\frac{1}{2} Z_{\rho}^{\prime}(0)$, which equals the logarithm of the Reidemeister-Franz torsion (W. Müller [3] and J. Cheeger [1]). A trace formula applied to flat manifolds plays a crucial role in our discussion (see [7], [10],[11],[12]).

\section{Trace formulae}

A compact orientable flat manifold $M$ is expressed as $M=\mathbb{R}^{n} / \Gamma$ with a torsion free discrete subgroup of the group of orientation preserving motions of $\mathbb{R}^{d}$. There is a natural one-to-one correspondence between the set of conjugacy classes $[\gamma], \gamma \in \Gamma$, and the set of free homotopy classes of maps of $S^{1}$ into $M$. We denote by $M_{[\gamma]}$ the set of closed geodesics $c: S^{1} \longrightarrow M$ belonging to the homotopy class $[\gamma]$. The space $M_{[\gamma]}$ equipped with compact open topology is a compact connected manifold, and the map $M_{[\gamma]} \longrightarrow M$ defined by $c \mapsto c(0)$ is an immersion which induces a flat metric on $M_{[\gamma]}$ (see [8], [9]). The fundamental group of $M_{[\gamma]}$ is isomorphic to the centralizer $\Gamma_{\gamma}$ of $\gamma$. We set $\ell_{\gamma}=$ length of $c \in M_{[\gamma]}$, which depends only on the class $[\gamma]$.

The following proposition is a straightforward generalization of the trace formula established in [7]. 
Proposition 2.1. Let $\Delta_{E}$ be the Laplacian acting on sections of the flat vector bundle $E$ associated with a representation $\rho: \Gamma \longrightarrow U(N)$. Then

$$
\operatorname{tr}\left(e^{-t \Delta_{E}}\right)=\sum_{[\gamma] \in[\Gamma]} \operatorname{tr} \rho(\gamma) \operatorname{vol}\left(M_{[\gamma]}\right) \alpha(\gamma)(4 \pi t)^{-\operatorname{dim} M_{[\gamma]} / 2} \exp \left(-\ell_{[\gamma]}^{2} / 4 t\right),
$$

where $\alpha(\gamma)=|\operatorname{det}(A(\gamma)-I \mid \operatorname{Im}(A(\gamma)-I))|^{-1}, A(\gamma)$ being the rotation part of the motion $\gamma$.

As an illustration, consider the case $M=S^{1}=\mathbb{R} / \mathbb{Z}$ and $\rho(n)=\exp 2 \pi \sqrt{-1} \alpha n$. The eigenvalues of $\Delta_{E}$ in this case are $4 \pi^{2}(n+\alpha)^{2}, n \in \mathbb{Z}$. Therefore the trace formula reduces to the classical summation formula

$$
\sum_{n \in \mathbb{Z}} \exp \left(-4 \pi^{2}(n+\alpha)^{2} t\right)=(4 \pi t)^{-1 / 2} \sum_{n \in \mathbb{Z}} \exp \left(2 \pi \sqrt{-1} n \alpha-n^{2} / 4 t\right),
$$

which is useful in later discussion.

Let $A: \Gamma \longrightarrow O(n)$ is the representation defined by taking the rotation part of each element in $\Gamma$. The $p$-th exterior product of the cotangent bundle, $\wedge^{p} T^{*} M$, is the flat bundle associated with the representation

$$
\wedge^{p} A: \Gamma \longrightarrow O\left(\wedge^{p} \mathbb{R}^{n}\right),
$$

so that the tensor product $E_{\rho} \otimes \wedge^{p} T^{*} M$ is the flat bundle associated with the representation $\rho \otimes \wedge^{p} A$. Applying the proposition above, we get

$$
=\sum_{[\gamma] \in[\Gamma]}^{\operatorname{tr}\left(e^{-t \Delta_{p}^{\rho}}\right)} \operatorname{tr} \rho(\gamma) \cdot \operatorname{tr}\left(\wedge^{p} A(\gamma)\right) \operatorname{vol}\left(M_{[\gamma]}\right) \alpha(\gamma)(4 \pi t)^{-\operatorname{dim} M_{[\gamma]} / 2} \exp \left(-\ell_{[\gamma]}^{2} / 4 t\right) .
$$

For brevity, we set

$$
\begin{aligned}
& D_{\rho}(t)=\sum_{p=0}^{n}(-1)^{p} p \operatorname{tr}\left(e^{-t \Delta_{p}^{\rho}}\right) \\
& =\sum_{[\gamma] \in[\Gamma]} \operatorname{tr} \rho(\gamma) \cdot\left(\sum_{p=0}^{n}(-1)^{p} p \operatorname{tr}\left(\wedge^{p} A(\gamma)\right)\right) \operatorname{vol}\left(M_{[\gamma]}\right) \alpha(\gamma) \\
& \times(4 \pi t)^{-\operatorname{dim} M_{[\gamma]} / 2} \exp \left(-\ell_{[\gamma]}^{2} / 4 t\right) .
\end{aligned}
$$

To transform $D_{\rho}$ still further, we divide the case into two parts.

(i) $n=2 k$. This being the case, the eigenvalues of $A(\gamma)$ are $e^{ \pm \sqrt{-1} \theta_{1}}, \ldots, e^{ \pm \sqrt{-1} \theta_{k}}$. Since $\operatorname{det}(I-x A)=\sum_{p=0}^{n}(-1)^{p} x^{p} \operatorname{tr}\left(\wedge^{p} A\right)$, we have

$$
\begin{aligned}
& \sum_{p=0}^{n}(-1)^{p} p \operatorname{tr}\left(\wedge^{p} A(\gamma)=\left.\frac{d}{d x}\right|_{x=1} \operatorname{det}(I-x A(\gamma))\right. \\
= & \left.\frac{d}{d x}\right|_{x=1} \prod_{i=1}^{k}\left(x^{2}-2 x \cos \theta_{i}+1\right)=k 2^{k} \prod_{i=1}^{k}\left(1-\cos \theta_{i}\right) .
\end{aligned}
$$

(ii) $n=2 k+1$. In this case, the eigenvalues of $A(\gamma)$ are $1, e^{ \pm \sqrt{-1} \theta_{1}}, \ldots, e^{ \pm \sqrt{-1} \theta_{k}}$. In the same way as (i), we find

$$
\sum_{p=0}^{n}(-1)^{p} p \operatorname{tr}\left(\wedge^{p} A(\gamma)\right)=-2^{k} \prod_{i=1}^{k}\left(1-\cos \theta_{i}\right) .
$$


We should note that, for any $\gamma \in \Gamma$, the kernel of $A(\gamma)-I$ is a non-zero vector subspace, and hence, in the case $n=2 k$, there exists some $i$ with $\theta_{i} \in 2 \pi \mathbb{Z}$. This implies $D_{\rho} \equiv 0$, and $Z_{\rho} \equiv 0$.

From now on, we shall confine ourselves to the case $n=2 k+1$. Note that $\prod_{i=1}^{k}\left(1-\cos \theta_{i}\right) \neq 0$ if and only if $\operatorname{dim} \operatorname{Ker}(A(\gamma)-I)=1$, or equivalently $\operatorname{dim} M_{[\gamma]}=$ 1. On the other hand, we have

$$
\alpha(\gamma)=\prod_{i=1}^{k}\left(2-2 \cos \theta_{i}\right)^{-1}
$$

Consequently we have

\section{Proposition 2.2.}

$$
D_{\rho}(t)=-\sum_{[\gamma]} \operatorname{tr} \rho(\gamma) \operatorname{vol}\left(M_{[\gamma]}\right)(4 \pi t)^{-1 / 2} \exp \left(-\ell_{[\gamma]}^{2} / 4 t\right),
$$

where $[\gamma]$ runs over all conjugacy classes with $\operatorname{dim} M_{[\gamma]}=1$.

Corollary 2.1. If $\operatorname{dim} M_{[\gamma]} \geq 2$ for every $[\gamma]$, then

$$
\sum_{p=0}^{n}(-1)^{p} p \operatorname{dim} H^{p}\left(M, E_{\rho}\right)=0 .
$$

\section{Primitive GeOdesics}

We will call a homotopy class $[\gamma]$ isolated if $\operatorname{dim} M_{[\gamma]}=1$. Intuitively speaking, this is equivalent to that there is no way to deform $c \in M_{[\gamma]}$ as geodesics except for changing the parameter of $c$.

Lemma 3.1. If $[\gamma]$ is isolated, then the ratio $\ell_{[\gamma]} / \operatorname{vol}\left(M_{[\gamma]}\right)$ is a positive integer.

Proof. Define the map $\widetilde{\omega}: S^{1} \longrightarrow M_{[\gamma]}$ by $\widetilde{\omega}(s)=c_{s}$, where $c_{s}(t)=c(s+t)$. If we equip $S^{1}$ with the metric induced from $c: S^{1} \longrightarrow M$, then $\widetilde{\omega}$ is a local isometry. Since $\operatorname{vol}\left(S^{1}\right)=\ell_{[\gamma]}$, and $\ell_{[\gamma]} / \operatorname{vol}\left(M_{[\gamma]}\right)$ equals the degree of the covering map $\widetilde{\omega}$, we are done.

A class $[\gamma]$ is said to be primitive if $[\gamma]$ is isolated and $\ell_{[\gamma]} / \operatorname{vol}\left(M_{[\gamma]}\right)=1$. The geometric meaning of this concept is the following: A closed geodesic is said to be prime if it is not an $m$-fold cover of another geodesic with $m>1$. Here we define the $m$-fold cover $c^{m}$ of $c$ by $c^{m}(t)=c(m t)$. A class $[\gamma]$ is primitive if and only if $[\gamma]$ is isolated and a geodesic $c \in M_{[\gamma]}$ is prime. One may also give a group theoretic meaning. A class $[\gamma]$ is isolated if and only if $\Gamma_{\gamma}$ is isomorphic to $\mathbb{Z}$. An isolated $[\gamma]$ is primitive if and only if $\gamma$ generates $\Gamma_{\gamma}$.

Lemma 3.2. (1) If $[\gamma]$ is primitive, then so is $\left[\gamma^{-1}\right]$.

(2) For any isolated class $[\gamma]$, there exist a unique primitive class $[\mu]$ and a positive integer $m$ such that $[\gamma]=\left[\mu^{m}\right]$.

Since (1) is obvious, we shall prove (2). Let $c \in M_{[\gamma]}$. There exist a unique prime closed geodesic $c_{1}$ and $m \geq 1$ with $c=c_{1}^{m}$. Suppose $c_{1} \in M_{[\nu]}$ (and hence $[\gamma]=$ $\left.\left[\nu^{m}\right]\right)$. The class $[\nu]$ is isolated because $1=\operatorname{dim} M_{[\gamma]} \geq \operatorname{dim} M_{[\nu]} \geq 1$. Here we have used the fact that the map $M_{[\nu]} \longrightarrow M_{\left[\nu^{m}\right]}=M_{[\gamma]}$ given by $c \mapsto c^{m}$ is an immersion. Next suppose that there is another primitive class $\left[\nu^{\prime}\right]$ with $\left[\left(\nu^{\prime}\right)^{m^{\prime}}\right]=[\gamma], m^{\prime} \geq 1$. 
Take $c^{\prime} \in M_{\left[\nu^{\prime}\right]}$. Then one can find some $s \in \mathbb{R}$ with $c^{\prime}\left(m^{\prime} t\right)=c(s+t), t \in \mathbb{R}$, which implies that $m=m^{\prime}$ and $c^{\prime}(t)=c_{1}(t+m s)$, so that $\left[\nu^{\prime}\right]=[\nu]$.

In view of the lemma above, one can find a set of primitive classes $\left\{\left[\mu_{\alpha}\right]\right\}_{\alpha \in A}$ such that any isolated class $[\gamma]$ can be written uniquely as $[\gamma]=\left[\mu_{\alpha}^{m}\right]$ for some $\alpha \in A$ and some $m \in \mathbb{Z}$. Noting that $\operatorname{vol}\left(M_{\left[\mu_{\alpha}^{m}\right]}\right)=\ell_{\left[\mu_{\alpha}\right]}$, we have

$$
D_{\rho}(t)=-(4 \pi t)^{-1 / 2} \sum_{\alpha \in A} \sum_{h \in \mathbb{Z}}{ }^{\prime} \operatorname{tr} \rho\left(\mu_{\alpha}^{h}\right) \ell_{\left[\mu_{\alpha}\right]} \exp \left(-h^{2} \ell_{\left[\mu_{\alpha}\right]}^{2} / 4 t\right)
$$

where, in the inner sum $\sum^{\prime}, h$ runs over all integers with isolated $\left[\mu_{\alpha}^{h}\right]$. From now on, we write $\ell_{\alpha}$ for $\ell_{\left[\mu_{\alpha}\right]}$. In order to describe such integers $h$, we let $\{1$, $\exp ( \pm$ $\left.\left.2 \pi \sqrt{-1} b_{\alpha 1} / a_{\alpha 1}\right), \ldots, \exp \left( \pm 2 \pi \sqrt{-1} b_{\alpha k} / a_{\alpha k}\right)\right\}$ be the eigenvalues of $A\left(\mu_{\alpha}\right)$, where $a_{\alpha j}, b_{\alpha j}(j=1, \ldots, k)$ are positive integers with $\left(a_{\alpha j}, b_{\alpha j}\right)=1$ (co-prime). Since $\operatorname{dim} \operatorname{Ker}\left(A\left(\mu_{\alpha}\right)-I\right)=1$, we have $a_{\alpha i}>1$. Note that $\left[\mu_{\alpha}^{h}\right]$ is isolated if and only if $a_{\alpha j}$ is not a divisor of $h$ for any $j=1, \ldots, k$. Therefore the "Inclusion-Exclusion Principle" leads us to

$$
\begin{aligned}
& \sum_{h \in \mathbb{Z}}{ }^{\prime} \operatorname{tr} \rho\left(\mu_{\alpha}^{h}\right) \exp \left(-h^{2} \ell_{\alpha}^{2} / 4 t\right) \\
= & \sum_{h \in \mathbb{Z}} \operatorname{tr} \rho\left(\mu_{\alpha}^{h}\right) \exp \left(-h^{2} \ell_{\alpha}^{2} / 4 t\right)-\sum_{m=1}^{k} \sum_{h \in \mathbb{Z}} \operatorname{tr} \rho\left(\mu_{\alpha}^{h a_{\alpha m}}\right) \exp \left(-h^{2} a_{\alpha m^{2}}^{2} \ell_{\alpha}^{2} / 4 t\right) \\
& +\sum_{1 \leq m_{1}<m_{2} \leq k} \sum_{h \in \mathbb{Z}} \operatorname{tr} \rho\left(\mu_{\alpha}^{h\left[a_{\alpha m_{1}}, a_{\alpha m_{2}}\right]} \exp \left(-h^{2}\left[a_{\alpha m_{1}}, a_{\alpha m_{2}}\right]^{2} \ell_{\alpha}^{2} / 4 t\right)-\cdots,\right.
\end{aligned}
$$

where the symbol $[p . q . r . \cdots]$ means the least common multiple of numbers p.q.r....

We now let $\left\{\exp 2 \pi \sqrt{-1} \theta_{\alpha 1}, \ldots, \exp 2 \pi \sqrt{-1} \theta_{\alpha N}\right\}$ be the eigenvalues of $\rho\left(\mu_{\alpha}\right)$. Substituting these values for $\operatorname{tr} \rho\left(\mu_{\alpha}^{h}\right)$, we obtain

$$
\begin{aligned}
D_{\rho}(t)= & -\sum_{\alpha \in A} \sum_{j=1}^{N} \sum_{u=0}^{k}(-1)^{u} \sum_{1 \leq m_{1}<\cdots<m_{u} \leq k}(4 \pi t)^{-1 / 2} \ell_{\alpha} \\
& \cdot \sum_{h \in \mathbb{Z}} \exp \left(2 \pi \sqrt{-1} \theta_{\alpha j} h\left[a_{\alpha m_{1}}, \ldots, a_{\alpha m_{u}}\right]-h^{2}\left[a_{\alpha m_{1}}, \ldots, a_{\alpha m_{u}}\right]^{2} \ell_{\alpha}^{2} / 4 t\right) .
\end{aligned}
$$

Here, for $u=0$, we understand $\left[a_{\alpha m_{1}}, \ldots, a_{\alpha m_{u}}\right]$ to be 1 . This is the stage to use the summation formula (2) to get

$$
\begin{aligned}
D_{\rho}(t)= & -\sum_{\alpha \in A} \sum_{j=1}^{N} \sum_{u=0}^{k}(-1)^{u} \sum_{1 \leq m_{1}<\cdots<m_{u} \leq k}\left[a_{\alpha m_{1}}, \ldots, a_{\alpha m_{u}}\right]^{-1} \\
& \cdot \sum_{h \in \mathbb{Z}} \exp \left(-4 \pi^{2} \frac{\left(h-\theta_{\alpha}\left[a_{\alpha m_{1}}, \ldots, a_{\alpha m_{u}}\right]\right)^{2} t}{\left[a_{\alpha m_{1}}, \ldots, a_{\alpha m_{u}}\right]^{2} \ell_{\alpha}^{2}}\right) .
\end{aligned}
$$

Proposition 3.1. There exist only finite many primitive classes.

Proof. In (5), we let $\rho$ be the trivial representation 1 . It should be noted that the series in the right hand side of (5) converges absolutely and each term is dominated by a positive $K_{\alpha j \ell m_{1} \cdots m_{\ell}}$, which does not depend on $t>0$ and satisfies $\sum K_{\alpha j \ell m_{1} \cdots m_{\ell}}<\infty$. Therefore, we may first take the limit $(t \uparrow \infty)$ of each term in 
the series, and find

$$
\lim _{t \rightarrow \infty} D_{\mathbf{1}}(t)=-\sum_{\alpha \in A} \sum_{u=0}^{k}(-1)^{u} \sum_{1 \leq m_{1}<\cdots<m_{u} \leq k}\left[a_{\alpha m_{1}}, \ldots, a_{\alpha m_{u}}\right]^{-1} .
$$

On the other hand, we have

$$
\lim _{t \rightarrow \infty} D_{\mathbf{1}}(t)=\sum_{p=0}^{n}(-1)^{p} p \operatorname{dim} \operatorname{Ker} \Delta_{p}=\sum_{p=0}^{n}(-1)^{p} p \operatorname{dim} H^{p}(M, \mathbb{R}) .
$$

We set, for a sequence of positive integers $a_{1}, \ldots, a_{k}$ with $a_{i}>1$,

$$
\left[\left[a_{1}, \ldots, a_{k}\right]\right]=\sum_{u=0}^{k}(-1)^{u} \sum_{1 \leq m_{1}<\cdots<m_{u} \leq k}\left[a_{m_{1}}, \ldots, a_{m_{u}}\right]^{-1} .
$$

Since, as shown below,

$$
\left[\left[a_{1}, \ldots, a_{k}\right]\right] \geq \prod_{i=1}^{k}\left(1-a_{i}^{-1}\right)\left(\geq 2^{-k}\right)
$$

and

$$
\lim _{t \rightarrow \infty} D_{\mathbf{1}}(t)=-\sum_{\alpha \in A}\left[\left[a_{\alpha 1}, \ldots, a_{\alpha k}\right]\right], \quad\left(a_{\alpha j}>1\right),
$$

the set $A$ is necessarily finite.

We shall prove (6) by induction on $k$. In the same time, we prove

$$
\left[\left[\theta_{1} a_{1}, \ldots, \theta_{k} a_{k}\right]\right] \geq\left[\left[a_{1}, \ldots, a_{k}\right]\right]
$$

for positive integers $\theta_{1}, \ldots, \theta_{k}$. For $k=1$, we have $\left[\left[a_{1}\right]\right]=1-a_{1}^{-1}$ and $\left[\left[\theta_{1} a_{1}\right]\right]=$ $1-\left(\theta_{1} a_{1}\right)^{-1} \geq 1-a_{1}^{-1}=\left[\left[a_{1}\right]\right]$. Suppose that our claim holds up to $k-1$. An easy computation leads us to

$$
\left[\left[a_{1}, \ldots, a_{k}\right]\right]=\left[\left[a_{2}, \ldots, a_{k}\right]\right]-\frac{1}{a_{1}}\left[\left[\frac{\left[a_{1}, a_{2}\right]}{a_{1}}, \ldots, \frac{\left[a_{1}, a_{k}\right]}{a_{1}}\right]\right] .
$$

Hence, noting $a_{i}=\frac{\left[a_{1}, a_{i}\right]}{a_{1}}\left(a_{1}, a_{i}\right)$, and using the induction hypothesis, we get

$$
\left[\left[a_{1}, \ldots, a_{k}\right]\right] \geq\left(1-a_{1}^{-1}\right)\left[\left[a_{2}, \ldots, a_{k}\right]\right] \geq \prod_{i=1}^{k}\left(1-a_{i}^{-1}\right) .
$$

We shall show (7). For this, it is enough to check $\left[\left[\theta a_{1}, a_{2}, \ldots, a_{k}\right]\right] \geq\left[\left[a_{1}, \ldots, a_{k}\right]\right]$. Using again (8), we obtain

$$
\begin{aligned}
{\left[\left[\theta a_{1}, a_{2}, \ldots, a_{k}\right]\right]-\left[\left[a_{1}, \ldots, a_{k}\right]\right]=} & \frac{1}{a_{1}}\left\{\left[\left[\frac{\left[a_{1}, a_{2}\right]}{a_{1}}, \ldots, \frac{\left[a_{1}, a_{k}\right]}{a_{1}}\right]\right]\right. \\
& \left.-\frac{1}{\theta}\left[\left[\frac{\left[\theta a_{1}, a_{2}\right]}{\theta a_{1}}, \ldots, \frac{\left[\theta a_{1}, a_{k}\right]}{\theta a_{1}}\right]\right]\right\} \\
\geq & \frac{1}{a_{1}}\left(1-\theta^{-1}\right)\left[\left[\frac{\left[a_{1}, a_{2}\right]}{a_{1}}, \ldots, \frac{\left[a_{1}, a_{k}\right]}{a_{1}}\right]\right] \geq 0
\end{aligned}
$$

where we have used that

$$
\frac{\left[a_{1}, a_{i}\right]}{a_{1}} / \frac{\left[\theta a_{1}, a_{i}\right]}{\theta a_{1}}=\frac{\left(\theta a_{1}, a_{i}\right)}{\left(a_{1}, a_{i}\right)}
$$

is a positive integer. 


\section{Corollary 3.1.}

$$
\begin{aligned}
& \sum_{p=0}^{n}(-1)^{p} p \operatorname{dim} H^{p}\left(M, E_{\rho}\right) \\
= & \sum_{\alpha \in A} \sum_{j=1}^{N} \sum_{u=0}^{k}(-1)^{u} \sum_{1 \leq m_{1}<\cdots<m_{u} \leq k}\left[a_{\alpha m_{1}}, \ldots, a_{\alpha m_{u}}\right]^{-1}\left\langle\theta_{\alpha j}\left[a_{\alpha m_{1}}, \ldots, a_{\alpha m_{u}}\right]\right\rangle,
\end{aligned}
$$

where the symbol $\langle\cdot\rangle$ denotes the characteristic function of $\mathbb{Z}$ in $\mathbb{R}$; that is, $\langle a\rangle=1$ if $a \in \mathbb{Z}$, otherwise $\langle a\rangle=0$. In particular

$$
\sum_{p=0}^{n}(-1)^{p} p \operatorname{dim} H^{p}(M, \mathbb{R})=-\sum_{\alpha \in A}\left[\left[a_{\alpha 1}, \ldots, a_{\alpha k}\right]\right] \leq 0 .
$$

\section{ZETA FUNCTIONS AND TORSIONS}

We are now in a position to calculate the Ray-Singer zeta function

$$
Z_{\rho}(s)=\Gamma(s)^{-1} \int_{0}^{\infty} t^{s-1}\left(D_{\rho}(t)-\lim _{t \rightarrow \infty} D_{\rho}(t)\right) d t
$$

In virtue of the identity

$$
\Gamma(s)^{-1} \int_{0}^{\infty} t^{s-1} \exp \left(-4 \pi^{2} \frac{(h-\alpha)^{2} t}{a^{2}}\right) d t=\left(\frac{a^{2}}{4 \pi^{2}}\right)^{s}(h-\alpha)^{-2 s} \quad(h \neq \alpha),
$$

we obtain

$$
\begin{aligned}
Z_{\rho}(s)= & -\sum_{\alpha \in A} \sum_{j=1}^{N} \sum_{\ell=0}^{k}(-1)^{\ell} \sum_{1 \leq m_{1}<\cdots<m_{\ell} \leq k}\left[a_{\alpha m_{1}}, \ldots, a_{\alpha m_{\ell}}\right]^{2 s-1}\left(\ell_{\alpha} / 2 \pi\right)^{2 s} \\
& \times \sum_{h \in \mathbb{Z}}^{\prime}\left(h-\theta_{\alpha j}\left[a_{\alpha m_{1}}, \ldots, a_{\alpha m_{\ell}}\right]\right)^{-2 s}
\end{aligned}
$$

where $h$ runs over integers with $h \neq \theta_{\alpha j}\left[a_{\alpha m_{1}}, \ldots, a_{\alpha m_{\ell}}\right]$. Therefore in terms of the Hurwitz zeta function $\zeta(s, \theta)$, we have

\section{Theorem 4.1.}

$$
\begin{aligned}
Z_{\rho}(s)= & -\sum_{\alpha \in A} \sum_{j=1}^{N} \sum_{u=0}^{k}(-1)^{u} \sum_{1 \leq m_{1}<\cdots<m_{u} \leq k}\left[a_{\alpha m_{1}}, \ldots, a_{\alpha m_{u}}\right]^{2 s-1}\left(\ell_{\alpha} / 2 \pi\right)^{2 s} \\
& \times\left\{\left\langle\theta_{\alpha j}\left[a_{\alpha m_{1}}, \ldots, a_{\alpha m_{u}}\right]\right\rangle 2 \zeta(2 s)\right. \\
& +\left(1-\left\langle\theta_{\alpha j}\left[a_{\alpha m_{1}}, \ldots, a_{\alpha m_{u}}\right]\right\rangle\right) \times\left(\zeta\left(2 s,\left\langle\left\langle\theta_{\alpha j}\left[a_{\alpha m_{1}}, \ldots, a_{\alpha m_{u}}\right]\right\rangle\right\rangle\right)\right. \\
& \left.\left.+\zeta\left(2 s, 1-\left\langle\left\langle\theta_{\alpha j}\left[a_{\alpha m_{1}}, \ldots, a_{\alpha m_{u}}\right]\right\rangle\right\rangle\right)\right)\right\}
\end{aligned}
$$

where $\langle\langle\alpha\rangle\rangle=\alpha-[\alpha]$.

The expression of $Z_{\rho}(s)$ above allows us to compute the Reidemeister-Franz torsion. Since

$$
\zeta(s, \theta)=\left(\frac{1}{2}-\theta\right)+\log \left(\frac{\Gamma(\theta)}{\sqrt{2 \pi}}\right) s+\cdots
$$


around $s=0$, we have

$$
\begin{aligned}
Z_{\rho}^{\prime}(0)= & -2 \sum_{\alpha \in A} \sum_{j=1}^{N} \sum_{u=0}^{k}(-1)^{u} \sum_{1 \leq m_{1}<\cdots<m_{u} \leq k}\left[a_{\alpha m_{1}}, \ldots, a_{\alpha m_{u}}\right]^{-1} \\
& \times\left\{-\left\langle\theta_{\alpha j}\left[a_{\alpha m_{1}}, \ldots, a_{\alpha m_{u}}\right]\right\rangle \log \left(\left[a_{\alpha m_{1}}, \ldots, a_{\alpha m_{u}}\right] \frac{\ell_{\alpha}}{2 \pi}\right)\right. \\
& +\left(1-\left\langle\theta_{\alpha j}\left[a_{\alpha m_{1}}, \ldots, a_{\alpha m_{u}}\right]\right\rangle\right) \log \left(\frac{1}{2 \pi} \Gamma\left(\left\langle\left\langle\theta_{\alpha j}\left[a_{\alpha m_{1}}, \ldots, a_{\alpha m_{u}}\right]\right\rangle\right\rangle\right)\right. \\
& \left.\left.\times \Gamma\left(1-\left\langle\left\langle\theta_{\alpha j}\left[a_{\alpha m_{1}}, \ldots, a_{\alpha m_{u}}\right]\right\rangle\right\rangle\right)\right)\right\},
\end{aligned}
$$

which, in view of the identity $\Gamma(x) \Gamma(1-x)=\frac{\pi}{\sin \pi x}$, leads us to

Theorem 4.2. The torsion $T_{\rho}(M)=\exp \left(\frac{1}{2} Z_{\rho}^{\prime}(0)\right)$ equals

$$
\begin{aligned}
& \prod_{\alpha \in A} \prod_{j=1}^{N} \prod_{u=0}^{k} \prod_{1 \leq m_{1}<\cdots<m_{u} \leq k} \\
& \left(\left[a_{\alpha m_{1}}, \ldots, a_{\alpha m_{u}}\right] \frac{\ell_{\alpha}}{2 \pi}\right)^{(-1)^{u}\left\langle\theta_{\alpha j}\left[a_{\alpha m_{1}}, \ldots, a_{\alpha m_{u}}\right]\right\rangle /\left[a_{\alpha m_{1}}, \ldots, a_{\alpha m_{u}}\right]} \\
& \times\left(2 \sin \pi\left\langle\left\langle\theta_{\alpha j}\left[a_{\alpha m_{1}}, \ldots, a_{\alpha m_{u}}\right]\right\rangle\right\rangle\right)^{(-1)^{u}\left(1-\left\langle\theta_{\alpha j}\left[a_{\alpha m_{1}}, \ldots, a_{\alpha m_{u}}\right]\right\rangle\right) /\left[a_{\alpha m_{1}}, \ldots, a_{\alpha m_{u}}\right]} .
\end{aligned}
$$

\section{REFERENCES}

[1] J. Cheeger, Analytic torsion and the heat equation, Ann. of Math. 109 (1979), 259-322.

[2] S. Kobayashi and K. Nomizu,Foundation of Differential Geometry, volume I, Interscience Publishers, 1969.

[3] W. Müller, Analytic torsion and R-torsion of Riemannian manifolds, Advances in Math. 28 (1978), 233-305.

[4] D. B. Ray and I. M. Singer, R-torsion and the Laplacian on Riemannian manifolds, Advances in Math. 7 (1971), 145-210.

[5] D. B. Ray, Reidemeister torsion and the Laplacian on lens spaces, Advances in Math. 7 (1970), 109-126.

[6] A. Selberg, Harmonic analysis and discontinuous groups in weakly symmetric spaces with applications to Dirichlet series, J. Indian Math. Soc. 20 (1956), 47-87

[7] T. Sunada, Spectrum of a compact flat manifold, Comment. Math. Helv. 53 (1978), 615-621.

[8] T. Sunada, Closed geodesics in a locally symmetric space, Tohoku Math.J. 30 (1978), 59-68.

[9] T. Sunada, Rigidity of certain harmonic mappings, Invent. Math. 51(1979), 297-307.

[10] T. Sunada, Trace formulas and heat equation asymptotics for a non-positively curved manifold, Amer. J. Math. 104 (1982), 795-812.

[11] T. Sunada, Trace formula, Wiener integrals, and asymptotics, in Proc. of the Franco-Japan Seminar, Kyoto 1981, (1983), 103-113.

[12] T. Sunada and M. Nishio, Trace formulae in spectral geometry, Proc. I.C.M. Kyoto 1990, Springer-Verlag Tokyo 1991, 577-585.

[13] H. Urakawa, Analytic torsion of space forms of certain compact symmetric spaces, Nagoya Math. J. 67 (1977), 65-88.

[14] J. A. Wolf, Spaces of Constant Curvature, McGraw-Hill, 1967.

Meiji Institute for Advanced Study of Mathematical Sciences, MeijI University

Graduate School of Information Science, Tohoku University 